\title{
Common polymorphisms in dystonia-linked genes and susceptibility to the sporadic
} primary dystonias

Jeremy R. B. Newman ${ }^{1}$, Greg T. Sutherland ${ }^{1,2}$, Richard S. Boyle ${ }^{3}$, Nicole Limberg ${ }^{3}$. Stefan Blum $^{3}$, John D. O’Sullivan ${ }^{4}$, Peter A. Silburn ${ }^{4,5}$, George D. Mellick ${ }^{1,4,5^{*}}$

1. Eskitis Institute for Cell and Molecular Therapies, Griffith University, Brisbane, Australia

2. Discipline of Pathology, Sydney Medical School, University of Sydney, Sydney, Australia

3. Department of Neurology, Princess Alexandra Hospital, Brisbane, Australia

4. Department of Neurology, Royal Brisbane and Women's Hospital, Brisbane, Australia

5. University of Queensland Centre for Clinical Research, School of Medicine, Royal Brisbane and Women's Hospital, Brisbane, Australia

*Corresponding author: Associate Professor George D. Mellick, Clinical Neuroscience Group, Eskitis Institute for Cell and Molecular Therapies, Griffith University, Don Young Road, Nathan QLD 4111, Australia, phone: +61 7373 55019, fax: + 617373 54255, E-mail: G.Mellick@griffith.edu.au

Key words: primary dystonia; polymorphism; case-control study; meta-analysis; GCH1; TOR1A

Short title: DYT SNPs and sporadic dystonia

Abstract: 203 words Text word count: 3000 words 


\section{Abstract}

Genes involved in familial dystonia syndromes (DYT genes) are ideal candidates for investigating whether common genetic variants influence the susceptibility to sporadic primary dystonia. To date, there have been few candidate gene studies for primary dystonia and only two DYT genes, TORIA and THAP1, have been assessed. We therefore employed a haplotypetagging strategy to comprehensively assess if common polymorphisms in eight DYT genes (TOR1A, TAF1, GCH1, THAP1, MR-1 (PNKD), SGCE, ATP1A3 and PRKRA) confer risk for sporadic primary dystonia. 230 primary dystonia cases were matched for age and gender to 228 controls, recruited from movement disorder clinics in Brisbane, Australia and the Australian electoral roll. All subjects were genotyped for 56 tagging SNPs and genotype associations were investigated. Modest genotypic associations $(P<0.05)$ were observed for three GCH1 SNPs (rs12147422, rs3759664 and rs10483639) when comparing all cases against controls. Associations were also seen when the cases were stratified based on presentation. Overall, our findings do not support the hypothesis that common TORIA variants affect susceptibility for sporadic primary dystonia, and that it is unlikely that common variants around the DYT genes confer substantial risk for sporadic primary dystonia. Further work is warranted to follow up the GCH1 SNPs and the subgroup analyses. 


\section{Introduction}

Primary dystonia is a group of related movement disorders characterised by abnormal repetitive, twisting postures due to the involuntary co-contraction of opposing muscle groups. This is thought to arise from a dysfunction of the central neural circuits that control and coordinate voluntary movements, such as those found in the basal ganglia [1], the cerebellum [2], the sensorimotor cortex [3], and the interactions between these three regions of the brain [4]. While the rare early-onset forms of dystonia can sometimes be traced to inherited genetic abnormalities, the more common late-onset forms arise sporadically with no clear aetiology [1]. It has been postulated that the sporadic forms of primary dystonia are caused by an interaction between genetic and non-genetic factors [5]. Sporadic primary dystonia is comprised of multiple phenotypic subtypes, classified by bodily presentation and distribution. However, the prevalence of primary dystonia is relatively low in the general population with the average European prevalence estimated at 152 cases per million and the estimated prevalence of each subtype substantially lower [6]. While there may be subtype-specific risk factors [7-9], there may also be susceptibility factors that are common to all sporadic primary dystonias. Given that the different subtypes can co-present in individual patients (concurrently at onset or sequentially) and that many seem to share neurophysiological similarities [5], it seems valid to group these subtypes into a single phenotype to identify such shared risk factors. The purpose of this study is to identify shared genetic risk factors for the sporadic primary dystonias.

To date there have been at least 17 genetic loci linked to familial forms of dystonia. At eight of these loci, causative mutations have been found in so-called DYT genes. These mutations cause rare and usually early-onset forms of familial dystonia or "dystonia plus" syndromes, including torsion dystonia (for example, DYT1, DYT6), myoclonus dystonia (DYT11), dystonia-parkinsonism (DYT3, DYT12, DYT16), paroxysmal/episodic dyskensia (DYT8) and dopa-responsive dystonia (DYT5) (for background information regarding these 
dystonia syndromes, please refer to Supplementary References). While these disorders display a rather disparate phenotypic spectrum dystonia is often prominent in these disorders, if not the only presenting symptom $[10,11]$. It is therefore an attractive option to investigate these genes as candidates for sporadic primary dystonia. This approach to candidate susceptibility loci has been employed with some success for other neurological diseases which have known genetic causes in a small proportion of cases; good examples include Parkinson's disease [12] and Alzheimer's disease [13].

To date there have been few published candidate genes association studies examining sporadic primary dystonia, and only two of the known DYT genes, TORlA (DYT1) and THAP1 (DYT6), have been studied. Six candidate genes studies have examined common single nucleotide polymorphisms (SNPs) in and around the TOR1A gene. Two of these studies showed strong associations between individual SNPs and susceptibility for primary dystonia in populations from Iceland [14], southern Germany and Austria [15], and the United States [16]. Others have shown modest associations in Indian, Italian and northern German populations [7, 8 , 17]. However, other similar studies have not replicated these findings $[8,18,19]$. In contrast to TORIA, no strong associations have been reported with polymorphisms in the THAPl gene [20, 21], although rare variants within the THAP1 coding regions may be a cause of some adultonset cases [22].

Here we looked to extend the previous studies by testing the hypothesis that common SNPs in various DYT genes influence the susceptibility to sporadic primary dystonia in a wellcharacterised Australian case-control sample. First, we used a similar haplotype-tagging approach to that of Kamm et al. [15] to cover the common genetic variability in TORIA followed by a meta-analysis of four previously studied TOR1A SNPs. We then applied haplotype-tagging approaches to investigate seven other DYT genes: TAF1 (DYT3), GCH1 
(DYT5), THAP1 (DYT6), MR-1 (DYT8, also known as PNKD), SGCE (DYT11), ATP1A3 (DYT12) and PRKRA (DYT16).

\section{Methods}

\subsection{Case ascertainment}

Subjects were recruited from neurology clinics in Brisbane, Australia between 2003 and 2011. Diagnosis of sporadic primary dystonia was made by an experienced movement disorder specialist using standard diagnostic criteria. Cases were defined as "sporadic" if they were negative for the TORIA $\triangle \mathrm{E} 302 / 303$ mutation and either had an onset age greater than 30 years, or reported no family history of dystonia or dystonia-plus syndromes at time of recruitment. Patients with focal, segmental, multifocal or generalised dystonia were included in this study.

A questionnaire was employed to ascertain clinical and epidemiological information from dystonia patients. Control subjects underwent similar data collection procedures and were excluded if screening suggested any possibility of movement or cognitive disorders. Informed consent was obtained from all participating subjects and human research ethical clearance was obtained from all participating institutions.

In total, there were 230 dystonia cases (mean age $=63 \pm 14$ years, 82 males/148 females, mean onset age $=46 \pm 15$ years) and 228 age- and gender-matched controls (mean age $=68 \pm$ 11 years, 84 males/144 females). For 206 dystonia cases we had comprehensive phenotype information. 140 patients had focal dystonia, 51 had segmented dystonia, 10 had multifocal dystonia and 5 had generalised dystonia. Supplementary Table 1 summarises the characteristics for the five most prevalent dystonia subtypes in the dystonia sample. These subtypes are inclusive of patients with multiple presentations and as such some patients were included in more than one subgroup. Blood was collected from all subjects and DNA extracted by standard methods. A comprehensive mutation screen of all genes known to be involved in familial forms 
of dystonia was not performed, however all dystonia cases were negative for the TORIA $\Delta \mathrm{E} 302 / 303$ mutation.

\subsection{SNP genotyping and analyses}

Genetic variables were selected to "tag" the vast majority of common genetic variation in the coding region of the TORIA, TAF1, GCH1, THAP1, MR-1 (PNKD), SGCE, ATP1A3 and PRKRA genes $\pm 5 \mathrm{~kb}$ (minor allele frequencies $\left.>10 \%, \mathrm{r}^{2}>0.90\right)$. "Haplotype-tagging" SNPs were derived from the HapMap project CEU population (Data Rel 28/phase II+III, August10, on NCBI B36 assembly (http://hapmap.ncbi.nlm.nih.gov/) using the Haploview software package running "Tagger” (www.broad.mit.edu/mpg/haploview/). All TOR1A SNPs previously genotyped in published case-control association studies of sporadic primary dystonia were also genotyped here [7, 8, 14-19] and, where possible, were used to complement or substitute tagging SNPs derived from HapMap data. Where complete coverage of a genetic region was not possible using HapMap data, SNPs were selected from the 1000 Genomes Project (http://www.1000genomes.org). In the case of THAP1, the polymorphisms c.-237_-236GA>TT, c. $71+9 \mathrm{C}>\mathrm{A}$ and c.71+126T $>\mathrm{C}$ were also genotyped.

Design and implementation of genotyping assays for selected SNPs was carried out by the Australian Genome Research Facility in Brisbane, using the MassARRAY genotyping platform (Sequenom, San Diego, USA). Genotyping reliability was verified by independently re-genotyping a random selection of $10 \%$ of the original sample and there were no conflicts. Over the 56 genotyping assays, 96.9\% of genotypes were successfully called. All statistical analyses were carried out in PLINK version 1.07 (http://pngu.mgh.harvard.edu/ purcell/plink/). The odds ratios (ORs) for genotype associations (additive model) were calculated using logistic regression with adjustment for age and gender. Stratified analyses were also performed between 
case groups for each of the four most prevalent dystonia subtypes in our sample and nondiseased controls.

\subsection{Meta-analysis}

Candidate gene studies for the primary dystonias were identified using web-based MEDLINE database searches (PubMed). The keywords "dystonia" and "polymorphism*" yielded 127 references in English, published between January 1966 and January 2011. Additional references were obtained by reviewing citations within these 127 articles. Inclusion criteria were: (1) they were case-control studies in which the cases were clinically diagnosed with having a primary focal or segmental dystonia and controls were neurologically healthy; (2) the genotype and/or allele frequencies were reported; and (3) included polymorphisms in the TORIA gene. No attempts were made to locate any unpublished studies, or those that were not available from online subscriptions or from institutional holdings. Genotype information from the current genotyping study was included in the meta-analyses.

Analysis was limited to SNPs that had been studied in three or more populations. Pooled data were analysed using the MIX program, version 1.7 (http://mix-for-meta-analysis.info/). ORs were calculated for each individual study prior to a combined analysis based on reported genotypic and allelic information. Pooled data were first analysed using a fixed-effects model calculated by the Mantel-Haenszel method, and visualised using forest plots $(P<0.05)$. Because this approach assumes homogeneity between studies, the potential presence of heterogeneity was calculated using a Cochran's Q statistic. Analyses with a high degree of heterogeneity (Q statistic $P<0.1$ ) were repeated using a random-effects model calculated as per the DerSimonian-Laird to adjust for between-study variability.

\section{Results}




\subsection{Tagging SNP selection}

46 tagging SNPs were selected from HapMap data and published literature. Since no HapMap SNPs around the THAP1 gene met our tagging SNP selection criteria, we included three previously identified polymorphisms (c.-237_-236GA>TT, c.71+9C>A, c.71+126T>C). To further characterise the common variation in the THAPl genomic region, we complemented these variants with six SNPs identified in CEU individuals (minor allele frequency $>4 \%$ ) as a part of the 1000 Genomes Project. In addition, one of the three HapMap-derived tagging SNPs for the TAF1 gene (rs5980758) failed quality control assessment during assay design. In an effort to compensate for this loss of coverage we sought to include two additional TAF1 SNPs (rs113203261 and rs5981112) selected from the 1000 Genomes Project, however only rs113203261 passed assay design and was genotyped. In total, 56 tagging SNPs covering the common genetic variation in and around the TOR1A, TAF1, GCH1, THAP1, MR-1 (PNKD), SGCE, ATPIA3 and PRKRA genes $( \pm 5 \mathrm{~kb})$ were selected. All SNPs conformed to HardyWeinberg equilibrium in control subjects $(P>0.05)$, with the exception of GCH1 SNPs rs2183081 $(P=0.008)$ and $\operatorname{rs} 7147286(P=0.04)$. These were excluded from further analysis.

\subsection{TORIA}

No significant associations were seen between TORIA SNPs and sporadic primary dystonia in our dataset $(P>0.05)$ (Table 2). Given that associations have been reported in other populations [7, 14-17] we decided to perform meta-analyses to improve power and see if our results were comparable with data from the seven published case-control association studies that have analysed TORIA SNPs. Four of these SNPs have been examined in multiple studies and were suitable for meta-analyses - rs1182, rs3842225, rs1801968 and rs2297693[7, 8, 14-19]. In the case of one study [16] allele counts were calculated from reported minor allele frequencies and group size. Significant between-study heterogeneity was observed within the meta-analyses 
for all SNPs except for rs1801968; these analyses were re-performed using a random-effects model to adjust for this variability. Overall, pooled allelic frequencies for each of the metaanalyses were not significantly different between cases and controls $(P>0.05)$ (Figure 2).

\subsection{TAF1, GCH1, THAP1, PNKD, SGCE, ATP1A3 and PRKRA}

In general, there were few differences in SNP frequencies between dystonia and control groups. Three GCH1 SNPs showed modest associations with primary dystonia. The frequencies of rs10483639 and rs3759664 were significantly higher in the controls (odd ratio $(\mathrm{OR})=0.697$ (0.511-0.949), $P=0.022 ;$ and $\mathrm{OR}=0.629(0.443-0.894), P=0.010$, respectively). For rs12147422, the alternative allele was more common in the cases $(\mathrm{OR}=1.702(1.141-2.537)$, $P=0.01$ ) (Table 2). No differences in genotype frequencies for the SNPs tagging the TAF1, THAP1, MR-1 (PNKD), SGCE, ATPIA3 and PRKRA genes were seen between cases and controls $(P<0.05)$ (Table 2). Associations between $G C H 1$ polymorphisms and cervical dystonia were observed when the case group was stratified based on the site of presentation $(P<$ 0.05) (Table 3). Further stratification of case groups provided little useful data due to the limited numbers of cases in each group. However, the raw data is included in Supplementary Table 2 for completeness. None of the reported associations would survive any moderate multipletesting adjustment.

\section{Discussion}

Mutations in the TORIA, TAF1, GCH1, THAP1, MR-1 (PNKD), SGCE, ATP1A3 and PRKRA genes have all been previously linked with familial dystonia syndromes. As associations between common variants in TORIA and THAPI and sporadic dystonia have been previously reported, it seemed reasonable to extend a candidate gene approach to all eight genes. There are other DYT genes such as SLC2A1 (DYT18) which could have been investigated as interesting 
candidates in this project for association with primary dystonia. However, we limited our study to those genes where mutations lead to dystonia as a prominent (if not the sole) presenting symptom. SLC2A1 mutations have been associated with the GLUT1 deficiency syndromes, which have a highly variable phenotype of which paroxysmal dystonia may only be one of a constellation of possible signs [23].

We employed a haplotype-tagging strategy to effectively cover the majority of common variability in the chosen genes to investigate associations with sporadic primary dystonia. A similar approach has been used to study TOR $1 A$ variants in a previous case-control association study [15]. Our analysis revealed few differences in SNP distribution between dystonia and control subjects. We did identify three SNPs in GCH1 associated with sporadic primary dystonia, although these associations would not survive an adjustment for multiple testing. Using a minor allele frequency of $10 \%$, our sample size had a statistical power of $90 \%$ to detect ORs of greater than 1.900 when not corrected for multiple testing. When correcting for 56 tests, only ORs greater than 2.410 would be deemed significant [24]. We estimate our cohort size would need to be at least three times larger $(n=770)$ in order to satisfy multiple testing corrections and remain statistically powerful (>90\%) [24].

GCH1 encodes the enzyme GTP cyclohydrolase I, which is involved in the synthesis of tetrahydrobiopterin (BH4). BH4 is a necessary cofactor in the synthesis of L-DOPA and, by extension, other neurotransmitters including dopamine. Deficiencies in this production are the key neurophysiological hallmark of DYT5 DOPA-responsive dystonia [25]. In addition, altered dopamine neurotransmission is thought to be a feature of sporadic primary dystonia (see [26] for a review). Genetic variation in $G C H 1$ has been associated with different levels of pain sensitivity and tolerance [27]. A "pain-protective" haplotype has been associated with reduced GCH1 expression and BH4 levels [28]. Our study included one of the SNPs of this haplotype (rs10483639) and the remaining SNPs are tagged by rs3759664 according to HapMap CEU 
data. Taken together, these findings lend credence to the hypothesis that primary dystonia may arise through changes in dopamine neurotransmission. Given that the depletion of striatal dopamine is also feature of Parkinson's disease (PD), it is tempting to suggest that primary dystonia may share a common molecular pathway with PD. Interestingly, a similar association between the three significant GCH1 SNPs (rs10483639, rs12147422 and rs3759664) and a PD case group recruited in Brisbane was observed in our cohort (Supplementary Methods, Supplementary Table 3). Meta-analyses of several genome-wide association studies show associations between other GCH1 variants tagged by these SNPs and PD [29].

Our analysis did not reveal strong associations in other DYT genes and susceptibility to sporadic primary dystonia. Furthermore, meta-analyses of four TORIA SNPs demonstrated that they were not susceptibility factors for sporadic primary dystonia. This suggests that these genes are unlikely to be major determinants of susceptibility to this disorder. As the sporadic primary dystonias are a phenotypically heterogenous group of movement disorders, subtype-specific genetic risk factors may exist. We found preliminary evidence to suggest that SNPs in GCH1 are associated with a specific dystonia subtype (Table 3). It is prudent to assume that these associations are simply spurious findings due to the small sample size. However, if replicated these results suggest that common variants of different DYT genes could confer susceptibility to individual dystonia subtypes. As such, these subtype-specific effects might be diluted by the phenotypic heterogeneity of a combined primary dystonia case group. While phenotype-specific genetic associations have not been widely explored in sporadic dystonia, associations between three TORIA SNPs and specific dystonia subtypes have been recently reported. Rs1182 was associated with blepharospasm [8] and rs1801968 was a risk factor for blepharospasm, cervical dystonia and writer's cramp [7], although a study published during the submission of this manuscript did not support these associations [30]. Additionally, rs3842225 was less frequent in patients with cervical dystonia and laryngeal dystonia compared with CEPH controls [16]. The 
genotype frequencies of several TORIA SNPs highly correlated to rs3842225 were slightly different between controls and patients with oromandibular or laryngeal dystonia in our study (Supplementary Table 2). However sample sizes for these stratified analyses are generally small. Nevertheless, such phenotype-specific studies appear warranted if sample size issues could be sufficiently resolved, possibly through collaborative efforts. These studies may also clarify some of the inconsistencies between SNP association studies, like that of TORIA, which may be in part due to the differences in the clinical composition of each study.

To our knowledge, this is the first study to analyse common variants in multiple DYT genes and the susceptibility to sporadic primary dystonia, and the first to demonstrate associations between $G C H 1$ SNPs and sporadic primary dystonia. It is also the first study to perform meta-analyses on TORIA SNPs in the context of sporadic primary dystonia. The advantages of our study are that we have covered the majority of common genetic variation around multiple DYT genes, have used a large study group and have examined the dystonia subtypes. The main limitation of our study is sufficient statistical power, especially for investigating subtype-specific genetic risk factors. In conclusion we suspect that common genetic variants around the DYT genes do not greatly confer susceptibility to sporadic primary dystonia. However, future work investigating $\mathrm{GCH} 1$ as a candidate gene as well as subtypespecific analyses is warranted. The rarity of dystonia as a phenotype means that such studies are likely to require collaborative studies.

\section{Acknowledgements}

We would like to thank Gerhard Siebert, Nadeeka Dissanayaka, Sarah Day, Coral Gartner, Madeline Minslow and Karen O’Maley for expert assistance, Sullivan and Nicolaides Pathology for assistance with blood collection, and Brett Chapman, Jennifer Templeton and 
Tamara Macdonald for assistance with SNP genotyping design and implementation. Financial support for this project was obtained from internal funding for G. D. Mellick.

Financial disclosures/conflict of interest:

The authors disclose no conflict of interest. 


\section{References}

[1] Fahn S, Bressman SB, Marsden CD. Classification of dystonia. Adv Neurol. 1998;78:1-

10.

[2] Teo JT, van de Warrenburg BP, Schneider SA, Rothwell JC, Bhatia KP.

Neurophysiological evidence for cerebellar dysfunction in primary focal dystonia. J Neurol Neurosurg Psychiatry. 2009;80:80-3.

[3] Quartarone A, Morgante F, Sant'angelo A, Rizzo V, Bagnato S, Terranova C, et al. Abnormal plasticity of sensorimotor circuits extends beyond the affected body part in focal dystonia. J Neurol Neurosurg Psychiatry. 2008;79:985-90.

[4] Argyelan M, Carbon M, Niethammer M, Ulug AM, Voss HU, Bressman SB, et al. Cerebellothalamocortical connectivity regulates penetrance in dystonia. J Neurosci. 2009;29:9740-7.

[5] Defazio G, Berardelli A, Hallett M. Do primary adult-onset focal dystonias share aetiological factors? Brain. 2007;130:1183-93.

[6] Epidemiological Study of Dystonia in Europe (ESDE) Collaborative Group. A prevalence study of primary dystonia in eight European countries. J Neurol. 2000;247:787-92. [7] Bruggemann N, Kock N, Lohmann K, Konig IR, Rakovic A, Hagenah J, et al. The D216H variant in the DYT1 gene: a susceptibility factor for dystonia in familial cases? Neurology. 2009;72:1441-3.

[8] Clarimon J, Brancati F, Peckham E, Valente EM, Dallapiccola B, Abruzzese G, et al. Assessing the role of DRD5 and DYT1 in two different case-control series with primary blepharospasm. Mov Disord. 2007;22:162-6.

[9] Defazio G, Berardelli A, Abbruzzese G, Lepore V, Coviello V, Acquistapace D, et al. Possible risk factors for primary adult onset dystonia: a case-control investigation by the Italian Movement Disorders Study Group. J Neurol Neurosurg Psychiatry. 1998;64:25-32. 
[10] Doheny DO, Brin MF, Morrison CE, Smith CJ, Walker RH, Abbasi S, et al. Phenotypic features of myoclonus-dystonia in three kindreds. Neurology. 2002;59:1187-96.

[11] Stefanova E, Djarmati A, Momcilovic D, Dragasevic N, Svetel M, Klein C, et al. Clinical characteristics of paroxysmal nonkinesigenic dyskinesia in Serbian family with Myofibrillogenesis regulator 1 gene mutation. Mov Disord. 2006;21:2010-5.

[12] Nalls MA, Plagnol V, Hernandez DG, Sharma M, Sheerin UM, Saad M, et al. Imputation of sequence variants for identification of genetic risks for Parkinson's disease: a meta-analysis of genome-wide association studies. Lancet. 2011;377:641-9.

[13] Guyant-Marechal L, Rovelet-Lecrux A, Goumidi L, Cousin E, Hannequin D, Raux G, et al. Variations in the APP gene promoter region and risk of Alzheimer disease. Neurology. 2007;68:684-7.

[14] Clarimon J, Asgeirsson H, Singleton A, Jakobsson F, Hjaltason H, Hardy J, et al. Torsin A haplotype predisposes to idiopathic dystonia. Ann Neurol. 2005;57:765-7.

[15] Kamm C, Asmus F, Mueller J, Mayer P, Sharma M, Muller UJ, et al. Strong genetic evidence for association of TOR1A/TOR1B with idiopathic dystonia. Neurology. 2006;67:1857-9.

[16] Sharma N, Franco RA, Jr., Kuster JK, Mitchell AA, Fuchs T, Saunders-Pullman R, et al. Genetic evidence for an association of the TOR1A locus with segmental/focal dystonia. Mov Disord. 2010;25:2183-7.

[17] Naiya T, Biswas A, Neogi R, Datta S, Misra AK, Das SK, et al. Clinical characterization and evaluation of DYT1 gene in Indian primary dystonia patients. Acta Neurol Scand. 2006;114:210-5.

[18] Hague S, Klaffke S, Clarimon J, Hemmer B, Singleton A, Kupsch A, et al. Lack of association with TorsinA haplotype in German patients with sporadic dystonia. Neurology. 2006;66:951-2. 
[19] Sibbing D, Asmus F, Konig IR, Tezenas du Montcel S, Vidailhet M, Sangla S, et al. Candidate gene studies in focal dystonia. Neurology. 2003;61:1097-101.

[20] Kaffe M, Gross N, Castrop F, Dresel C, Gieger C, Lichtner P, et al. Mutational screening of THAP1 in a German population with primary dystonia. Parkinsonism Relat Disord, published online, 22 July 2011 DOI: 10.1016/j.parkreldis.2011.06.023

[21] Xiao J, Zhao Y, Bastian RW, Perlmutter JS, Racette BA, Tabbal SD, et al. The c.237_236GA>TT THAP1 sequence variant does not increase risk for primary dystonia. Mov Disord. 2011;26:549-52.

[22] Xiao J, Zhao Y, Bastian RW, Perlmutter JS, Racette BA, Tabbal SD, et al. Novel THAP1 sequence variants in primary dystonia. Neurology. 2010;74:229-38.

[23] Brockmann K. The expanding phenotype of GLUT1-deficiency syndrome. Brain Dev. 2009;31:545-52.

[24] Menashe I, Rosenberg PS, Chen BE. PGA: power calculator for case-control genetic association analyses. BMC Genet. 2008;9:36.

[25] Furukawa Y. GTP Cyclohydrolase 1-Deficient Dopa-Responsive Dystonia. 1993.

[26] Peterson DA, Sejnowski TJ, Poizner H. Convergent evidence for abnormal striatal synaptic plasticity in dystonia. Neurobiol Dis. 2011;37:558-73.

[27] Campbell CM, Edwards RR, Carmona C, Uhart M, Wand G, Carteret A, et al. Polymorphisms in the GTP cyclohydrolase gene $(\mathrm{GCH} 1)$ are associated with ratings of capsaicin pain. Pain. 2009;141:114-8.

[28] Antoniades C, Shirodaria C, Van Assche T, Cunnington C, Tegeder I, Lotsch J, et al. GCH1 haplotype determines vascular and plasma biopterin availability in coronary artery disease effects on vascular superoxide production and endothelial function. J Am Coll Cardiol. 2008;52:158-65. 
[29] Lill CM, Roehr JT, McQueen MB, Kavvoura F, Bagade S, Schjeide BMM, et al. The PDGene Database. Alzheimer Research Forum. [cited 29 July 2011]; Available from: http://www.pdgene.org/.

[30] Chen Y, Burgunder JM, Song W, Huang R, Shang HF. Assessment of D216H DYT1 polymorphism in a Chinese primary dystonia patient cohort. Eur J Neurol, published online, 8 Nov 2011 DOI: 10.1111/j.1468-1331.2011.03582.x 


\section{Figures}

Figure 1. Genomic features of the TOR1A, TAF1, GCH1, THAP1, MR-1 (PNKD), SGCE, ATP1A3 and PRKRA genes. Transcripts for each gene were derived from HapMap data (obtained from NCBI B36 assembly). LD maps $\left(\mathrm{r}^{2}\right)$ were generated from all subjects in this study and visualised in Haploview.

Figure 2. Forest plot and meta-analyses of TORIA SNPs. Solid blocks and horizontal lines indicate the logarithmically scaled ORs and 95\% confidence intervals (CIs) respectively. Overall result of the meta-analysis is shown as the diamond. 